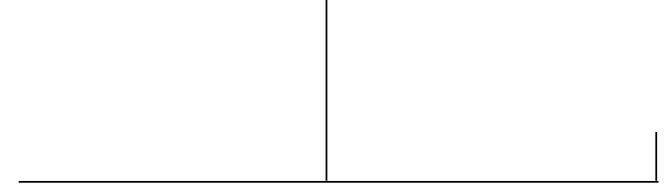

Rev. Latinoam. Psicopat. Fund., São Paulo, v. 12, n. 1, p. 99-115, março 2009

\title{
Jacques Lacan vers le réel (1936-1962)*
}

Fréderic Pellion

\begin{abstract}
Davantage que celles de "symbolique" et d' "imaginaire", la construction par Jacques Lacan de sa catégorie du "réel” n'est pas allée sans difficultés et sans repentirs. C'est de ces difficultés, et de quelques étapes de leur position puis de leur résolution, dont il sera question ici; non sans espérer, au terme du parcours, pouvoir peutêtre un peu mieux situer le rôle de cette catégorie dans la "métapsychologie" lacanienne - surtout si l'on admet que celle-ci n'est pas sans interréagir avec les métapsychologies voisines, en plus qu'avec de nombreux aspects du "dire" philosophique.
\end{abstract}

Mots clés: Impossible, objet a, sujet, vérité

* Ce texte est la réécriture de deux interventions au Collège Clinique Psychanalytique du Sud-Ouest, Bordeaux, le 29 mars 2008, et au séminaire du Master Recherche Médecine scientifique, psychopathologie et psychanalyse animé par Fethi Benslama, Université Paris VII - Denis Diderot, Paris, le 15 avril 2008. 
La réalité est (...) ce qui fonctionne, fonctionne vraiment. Mais ce qui fonctionne vraiment n'a rien à faire avec ce que je désigne $d u$ réel. C'est une supposition tout à fait précaire que mon réel (...) conditionne la réalité (...). Il y a là un abîme, dont on est loin de pouvoir assurer qu'il se franchit. (...) L'instance du savoir que

Freud renouvelle, je veux dire rénove sous la forme de l'inconscient, ne suppose pas du tout obligatoirement le réel dont je me sers. (...) C'est dans la mesure où Freud a vraiment fait une découverte - à supposer que cette découverte soit vraie - que l'on peut dire que le réel est ma réponse symptomatique. (...) La distinction du réel par rapport à la réalité, je ne suis pas sûr que ça se confonde avec la valeur propre que je donne au terme de réel.
\end{abstract}

Jacques Lacan, 1975-76, p. 132-135

Bercés par la mélodie ternaire du séminaire RSI, et plus généralement des nœuds borroméens, nous avons peut-être fini par prendre de fâcheuses habitudes: celle de tenir les trois registres lacaniens du réel, du symbolique et de l'imaginaire comme des acquis également indiscutables; et celle de les considérer comme en un certain sens interchangeables - ne serait-ce que par cette commune indiscutabilité. Deux habitudes certes pacifiantes, mais qui tendent, à mon avis, à occulter ce qu'il en est avant tout de leur foncière dyssymétrie.

Réexaminer la chronologie de leur construction ne laisse par contre plus douter de cette dyssymétrie: symbolique et imaginaire sont bien premiers dans 1' "ordre" lacanien "des raisons" (Guéroult). De la même manière que la distinction de la pensée et de l'étendue est inaugurale pour René Descartes, la question de leur "union" par une troisième substance n'étant affrontée que beaucoup plus tard, c'est en effet la distinction entre ces deux-là qui inaugure l'enseignement de Jacques Lacan - occupant, concrètement, les deux premières années du Séminaire (Lacan, 19531954, 1954-1955). La spécification du réel ne vient qu'ensuite, et sur le fond de cette distinction.

Il convient à mon avis de ne pas oublier cela si on veut apprécier correctement la fonction et l'économie de ce registre du réel dans la "pensée" (Lacan, 1969, p. 377) lacanienne de la psychanalyse. 
Très tôt, c'est-à-dire bien avant le début du Séminaire, Lacan semble bien avoir eu une appréhension intuitive de ce qui sera substantivé, par la suite, comme le réel. On est donc fondé à se demander le rôle de cette intuition dans les catégorisations ultérieures, y compris celles du symbolique et de l'imaginaire. Mais, d'abord, quelle est exactement sa teneur? Le futur réel est intialement étranger à la distinction entre symbolique et imaginaire qui n'a pas commencé sa carrière; il s'introduit plutôt par une différenciation avec autre chose, à savoir la "réalité".

Celle-ci est, avec le "moi", une des pierres d'angle de la psychanalyse des années $1930,{ }^{1}$ et c'est donc aussi comme objection à ces deux notions à l'époque solidairement reçues que cette différenciation doit être lue. Cette objection, de fait, se porte sur au moins deux fronts: celui de la méthode, car il s'agit rendre leur dignité clinique à des phénomènes que le principe de réalité, dominant, voudrait bien ne compter que comme des artefacts à patiemment réduire; celui de l'épistémè, puisqu'elle ambitionnne rien de moins que de refonder la théorie sur ces phénomènes - pour achever le geste du premier Freud, dont la postulation de pensées formées comme telles quoiqu'inconscientes ne peut que susciter un autre sujet que le sujet philosophique traditionnel, soit le sujet "supposé par la connaissance des objets" (Lacan, 1958-1959, leçon du 10 décembre 1958).

Ainsi, dès 1936, et dans un article justement intitulé "Au-delà du principe de réalité", Lacan, cherchant à cerner le type d' "objectivité" qui spécifierait l' "expérience analytique", a cette phrase: "L'image [suscitée par le transfert] est tout d'abord assimilée au réel, pour être ensuite désassimilée $d u$ réel et rendue à sa réalité propre" (p. 85, je souligne). On pressent déjà que ce "propre" fait le lit d'une "réalité" inégale au "réel" ordinaire, et qui renvoie à sa chimère l'objectif assumé par l'egopsychology d'une adaptation à la "réalité"...

En 1951, la même idée est reformulée en des termes qui déplacent ceux utilisés en 1936: "Le transfert n'est rien de réel dans le sujet, sinon l'apparition, dans un moment de stagnation de la dialectique analytique, des modes permanents selon lesquels il constitue ses objets" (p. 225, je souligne).

On pourrait commenter très longuement cette expression "rien de réel". 1) Elle est d'abord un bel exemple d'équivocité. En effet, à première vue, elle dit sim-

1. La première édition de l'ouvrage d'Anna Freud (Freud, Anna.) date de 1936, tout comme les premières communications de Heinz Hartmann sur la "psychologie du moi" (cf. Hartmann). 
plement - simplement, c'est-à-dire selon la doxa freudienne - que le transfert n'est aucunement, absolument pas, réel; l'empan du signifiant "réel" y est alors cantoné à celui de l'épithète communément accolée par la langue à la réalité. 2) Mais ce "rien de réel" évoque aussi ${ }^{2}$ - surtout si on lit la phrase jusqu'à son bout, bout où elle parle de la relation d'objet, voire du choix d'objet - un frayage à explorer entre ce qui deviendra "le" réel et cette figure limite de l'objectivité, classique depuis Blaise Pascal (Pascal), qu'est "le" rien - lequel "rien" deviendra par la suite pour Lacan, ${ }^{3}$ comme on le sait, une figure spécialement éloquente, car spécialement dématérialisée, ${ }^{4}$ de l'objet $a$.

Dans la conférence de juillet 1953 connue sous le titre "Le symbolique, l'imaginaire et le réel", le réel-substantif, quoiqu'annoncé, est quelque peu passé sous silence. Et Lacan, interrogé sur ce silence, a, avant de renvoyer au silence de l'analyste auquel le patient de "heurte", cette phrase assez énigmatique: "Le réel est ou la totalité, ou l'instant évanoui" (Lacan, 1953). "Totalité", "instant évanoui": deux figures encore indistinctes, aurais-je envie de dire, de l'immixtion - qui est aussi, considérée du point de vue du sujet, Lacan y reviendra beaucoup plus tard, donation $^{5}$ - du transcendant dans l'immanent; soit deux figures de la compacité prêtée à l'Autre dès lors qu'il se laisse envisager comme séparé.

La première réinterprête bien sûr le "sentiment océanique" freudien (Freud, 1921), et, si l'on oublie pas que la seconde, cet "instant évanoui", renvoie luimême à la "totalité" de par la plénitude existentielle qui lui est attribuée par le souvenir nostalgique, et/ou par la construction analysante, on voit tout de même s'esquisser le cahier des charges que le réel lacanien aura à remplir: faire coïncider, un peu comme maximum et minimum coïncident chez Nicolas de Cuse

2. Un peu comme dans le syntagme "bleu ciel", où on ne sait plus très bien si la substance du ciel est entièrement adsorbée par la détermination générique du bleu, ou bien s'il elle conserve une certaine individualité de référer à la couleur de tel ou tel ciel particulier, quitte à ce que $c e$ ciel soit impossible à retrouver dans le bleu ainsi qualifié.

3. Devenir préparé par: dans le Séminaire $I V$, l'inversion positivante du "ne rien manger" en "manger rien" (Lacan, 1956-1957, p. 184 sqq.); dans "L'instance de la lettre", la mise en valeur de l'intervention du "rem" latin dans la composition linguistique de la chose-res (Lacan, 1957, p. 495 sqq.); dans "La direction de la cure", la réédition de la manœuvre linguistique du Séminaire IV, lisant cette fois un "voler rien" latent derrière le "ne rien voler" manifeste, pour faire valoir finalement la demande comme "demande de rien" (Lacan, 1961, p. 620 et 627 sqq.).

4. Sur ce terme, $c f$. infra.

5. En particulier lorsque, dans le Séminaire XVI, il parlera de "tout donné" à propos de l'objet $a$ (Lacan, 1968-1969, p. 365). 
(Cuse), la marque ineffaçable du perçu et les différentes modalité de "perte de la réalité" (Freud, 1924a, 1924b) qui maintiennent, envers et contre tout, l'exquisité de cette marque. Il s'agit donc déjà, en somme, de demander au réel de prendre en charge une paradoxalité.

À cet égard, remarquons seulement trois choses: 1) la prise au sérieux de cette paradoxalité est cohérente avec la définition freudienne de la "réalité psychique": "La psychanalyse nous a exhortés à abandonner l'opposition infructueuse entre facteurs externes et internes, entre destin et constitution, et nous a enseigné à trouver régulièrement la causation de la maladie névrotique dans une situation psychique déterminée, laquelle peut être instaurée par des voies diverses" (Freud, 1912, p. 126);2) c'est d'ailleurs dans le prolongement direct du texte freudien que se situe, dans le séminaire sur Le moi..., l'excursus au cours duquel Lacan (1954-55) s'essaie à une première définition du réel: "Cette distinction [de l'extériorité et de l'intériorité] n'a aucun sens au niveau du réel. Le réel est sans fissure" (p. 122); 3) et enfin, qu'en 1951, l' "espace transitionnel” winnicottien (Winnicott) était déjà venu objecter à ce que la réalité formatrice de la psychè se superpose en tous points avec la réalité commune.

Si l'on excepte le bref passage du Séminaire II cité à l'instant, le "réel", comme "registre" proprement lacanien, apparaît à la page 50 du séminaire sur Les psychoses. ${ }^{6}$ Voici le passage considéré:

L'objet humain (...) n'est dépendant de la préparation d'aucune coaptation instinctuelle du sujet. (...) Le premier abord que [le sujet humain désirant] a de l'objet, c'est l'objet en tant qu'objet du désir de l'autre. Cela définit, à l'intérieur du rapport à la parole, quelque chose qui provient d'une autre origine - c'est exactement la distinction de l'imaginaire et du réel. Une altérité primitive est incluse dans l'objet, en tant qu'il est primitivement objet de rivalité et de concurrence. (...) Cette base rivalitaire et concurrentielle au fondement de l'objet est précisément ce qui est surmonté par la parole en tant qu'elle intéresse le tiers. (...) Cette

6. On peut toutefois considérer que ce passage au substantif se prépare vers la fin du séminaire de l'année précédente: "L'ombilic du rêve, cette relation abyssale au plus inconnu, qui est la marque d'une expérience privilégiée exceptionnelle, où un réel est appréhendé au-delà de toute médiation, qu'elle soit imaginaire ou symbolique" (Lacan, 1954-1955, p. 209, je souligne). 
distinction de l'Autre avec un grand A, c'est-à-dire de l'Autre en tant qu'il n'est pas connu, et de l'autre avec un petit a, c'est-à-dire de l'autre qui est moi, source de toute connaissance, est fondamentale. (Lacan, 1955-56, p. 50-51, je souligne).

Deux remarques me semblent nécessaires ici. 1) "Le" réel s'y distingue de "l"" imaginaire comme la "reconnaissance" dépasse et transcende la seule "connaissance". Cette opposition, toute abstraite qu'elle semble, est finalement, je pense, assez simple: dans le petit autre auquel nous avons affaire, est-ce d'abord le sujet ou l'objet que nous voyons? Dans le premier cas, il s'agit de reconnaissance, dans le second, de connaissance. 2) Mais cette distinction ne s'assure que de la référence à la topologie ambiguë (cf. les membres de phrase soulignés) que "le" réel suscite à cette parole qui est le détour obligé de la reconnaissance.

Ce pourquoi, le réel, pour devenir une dimension véritablement autonome, a aussi à s'affranchir de la tutelle d'un symbolique si essentiel à la première distinction, du réel et de l'imaginaire. Cet affranchissement, urgent dès lors que cette première distinction est posée, est amené par Lacan (1955-56) aux pages 94-95 du même séminaire:

Dans le rapport du sujet au symbole, il y a la possibilité d'une Verwerfung primitive, à savoir que quelque chose ne soit pas symbolisé, qui va se manifester dans le réel. La catégorie du réel est essentielle à introduire, elle est impossible à négliger dans les textes freudiens. Je lui donne ce nom en tant qu'elle définit un champ différent du symbolique.

De même, donc, que l'Ics est ab initio distingué du Cs eu égard au refoulement, le réel est donc défini à partir du mécanisme différent qu'est la forclusion. Mais notons - d'autant que Freud lui-même avait fini par ménager la possibilité d'un "refoulement originaire" [Urverdrängt] sans "retour" possible (Freud, 1915, p. 191-192) - que l'empan de ce mécanisme n'est pas a priori limité à la phénoménologie des psychoses: Lacan parle d'ailleurs du "rapport du sujet au symbole", et non du "rapport du sujet psychotique au symbole"...

À partir de ces deux distinctions, le Séminaire III et surtout la "Question préliminaire..." (Lacan, 1958a) commencent de relire autrement la différence initiale entre réalité et réel: le réel ne s'obtient pas par soustraction de la réalité, il n'en est pas le résidu, la "part maudite": il en est au contraire le principe organisateur, quoique ce principe lui soit en même temps foncièrement excentré, dysharmonique, ou, pour le dire encore une fois en termes freudiens, étranger (Freud, 1919b).

L'idée winnicottienne selon laquelle la réalité est, en quelque sorte, secrétée par le sujet au moins autant que par le Nebenmensch, est ainsi fait sienne par Lacan: on peut relire, à cet égard, la note ajoutée en 1966 à la "Question prélimi- 
naire", posant la constitution du champ de la réalité comme conséquence de l' "extraction de l'objet $a$ " (Lacan, 1958a, p. 553-554, n. 1), soit d'une opération du sujet sur le réel.

D'où, s'agissant de commenter Schreber se raccrochant à la fixité des étoiles, la difficulté de donner son statut à "ce qui revient toujours à la même place": est-ce déjà le réel, ou encore la réalité (Lacan, 1955-1956, p. 66)? À certains égards, en effet, le réel hallucinatoire participe d'un retour du même, de l' "immodifiable", 7 dès lors que l'on peut affirmer comme une règle générale que l'hallucination surgit là où l' "objet indicible est rejeté dans le réel" (Lacan, 1958a, p. 535). Notons seulement que, si l'objet "indicible" peut être rejeté au même titre que le signifiant non inscrit, "jamais venu à la place de l'Autre" (Lacan, 1958a, p. 577), c'est que la forclusion, comme mécanisme propre au réel, a, en quelque sorte, deux faces indissociables, l'une signifiante, l'autre objectale.

\section{II}

\section{Dématérialiser le réel}

Mais ce "rejet" d'un objet - au demeurant déjà envisagé par Freud comme cause ultime de l'affection mélancolique ${ }^{8}$-, est aussi dématérialisation de celuici. En effet, il ne reste de la matérialité perceptive de l'objet rejeté de la réalité que la forme signifiante selon laquelle il se re-matérialise. ${ }^{9}$ Cette dématérialisation du réel, ou plutôt cette dématérialisation qui fait passer de la réalité au réel, ou de l'objet au signifiant, va dès lors cheminer parallèlement à la "déchéance" (Lacan, 1966, p. 720) de l'imaginaire qui se joue à partir du Séminaire $I V$.

Il me semble ainsi que cette dématérialisation commence de prendre pour Lacan toute sa portée lorsque se consomme le divorce entre le " partenaire" érotique et la "cause du désir" (Lacan, 1958b, p. 691). On peut prendre pour exemple de ce mouvement la leçon du 7 janvier 1959 du séminaire sur Le désir et son

7. Jules Cotard a employé ce terme, et l'expression de "réalité immodifiable", pour qualifier ce que voudrait nier la "toute-puissance mélancolique" (Cotard).

8. Verworfene Objekt, lit-on ainsi, en 1921, sous la plume de Freud (Freud, 1921, p. 72).

9. Ce pourquoi cette dématérialisation est aussi réalisation, au sens où Roland Gori emploie ce terme (d'aucuns préféreraient le néologisme "réélisation", peut-être) à propos de la passion haineuse (Gori, 2005, p. 102 sqq.). 
interprétation, où Lacan clôt (provisoirement) les réflexions sur le fantasme amorcées deux ans auparavant ${ }^{10}$ en formulant que "ce qui le sujet a en lui-même comme pulsion est supporté par le partenaire petit $a$ " (Lacan, 1958-1959, leçon du 7 janvier 1959). Cette formule non seulement écrit la mise en cause propre au fantasme de la distinction sujet/objet, mais de surcroît parachève la mise en cause économique de la réalité par ce dernier: en effet, à partir d'elle, l' "objet du désir" - en quelque sorte refendu entre "pulsion", d'une part, et "partenaire petit $a$ ", de l' autre -, a définitivement plus de valeur que $i$ ' $(a)$ quant à la satisfaction "réelle" du sujet.

À partir de cette formule, les chemins de la dématérialisation du réel et de la décrédibilisation du fantasme - décrédibilisation nécessaire que Lacan épinglera ultérieurement d'un "chavirage" de "l' assurance" prise en lui (Lacan, 1968) -, confluent l'un avec l'autre. La croyance au perceptum du fantasme, en effet, est aussi croyance en une réalité qui aurait été cause - je vais y revenir.

Mais cet acmé du 7 janvier 1959 est suivi d'un suspens de l'élaboration: les cinq leçons qui suivent sont assez confuses, comme on peut le vérifier à la lecture, et le séminaire ne reprend véritablement son cours que par le commentaire de Hamlet qui occupe les sept leçons de mars et avril 1959. Or, soulignons que si, selon la très heureuse expression de Lacan, Hamlet est "tragédie du désir" (Lacan, 1958-1959, leçon du 11 mars 1959, Ornicar?, n. 24, p. 24, 1981), c'est sur le fond d'un véritable trop plein de "partenaires" que ce joue cette tragédie ${ }^{11}$ — car, justement, aucun de ses partenaires n'a le pouvoir de relancer le désir du prince.

C'est selon moi la même difficulté à dématérialiser le réel qui motive le "détour par la Chose" (Pellion, 2004-2007) du séminaire sur L'éthique de la psychanalyse. En effet, ce détour apparaît bien comme une sorte de régression des propositions du séminaire sur Le désir vers l'hypothèque substantialiste traditionnelle: ${ }^{12}$ si le désir est, alors sa cause ne peut être rien d'autre qu'un étant.

Mais les premières leçons de ce séminaire amènent dans le même temps une précision décisive quant à la définition du réel en posant que le problème moral

10. La leçon du 16 janvier 1957 du Séminaire $I V$ a été consacrée à mettre en évidence 1' "objectivation des signifiants" dont procède le fantasme freudien (cf. Freud, 1919a, et Lacan, 1956-1957, p. 113-121), puis, à la lumière de cette objectivation, les leçons du 12 février et du 26 mars 1958 du Séminaire $V$ ont interrogé les conséquences de l'anonymat de l'objet du fantasme (cf. Lacan,1957-1958, particulièrement p. 243 et 303).

11. Énumérons rapidement ces partenaires: côté masculin, il y a le père, le ghost, Claudius, sans oublier l'ami Horatio qui sera chargé à la toute fin de la pièce de rien de moins que de faire passer l'histoire à la postérité; côté féminin, Ophélie, bien sûr, mais aussi, derrière celle-ci, Gertrude, tous personnages avec lesquels Hamlet entretient une relation pour le moins animée. 12. Cf. infra. 
touche à celui-ci là où nous sommes incapables d'en donner une solution clairement articulée. ${ }^{13}$ Et c'est très exactement cette qualité-ci du réel - de se mettre en travers de l'enchaînement empirique perception $\rightarrow$ déduction - qui va être secondairement transférée à l'objet $a,{ }^{14}$ dès lors que celui-ci sera assumé par Lacan comme réel.

\section{Le réel, c'est l'impossible}

Cette formule, qui vaut comme spécification positive du réel au-delà de sa seule "dématérialité", se prépare, comme on vient de l'entrapercevoir, dès les premières leçons du Séminaire sur L'éthique. ${ }^{15}$ Elle attendra néanmoins encore quelques années pour se trouver formulée en toutes lettres, en 1964 (Lacan, 1964a, p. 152).

Mais le thème qu'elle recouvre suscite Lacan à toute une série de variations. Je vais ici en amplifier rapidement quatre: les deux premières relisant dans l'aprèscoup des énoncés antérieurs, les deux suivantes renvoyant plus précisément à l'intervalle de temps durant lequel le thème cherche sa formule.

Première variation: l'objet indicible comme cause. Revenons un peu en arrière. En effet, qu' est-ce que l' "objet indicible"? On peut dire, il me semble, qu'il est pure causalité: rien ne peut en être encore valablement compris comme sens, sauf qu'il est objet, c'est-à-dire cet autre au sujet qui provoque ce dernier, à l'instar de l'objet cartésien de l'admiration, à la possibilité de faire référence. ${ }^{16} \mathrm{Il}$ occupe donc, comme Bedeutung sans aucun Sinn, le champ laissé en jachère par le "phallicisme" (Lacan, 1956-1957, p. 29) analytique.

Seconde variation: l'agent comme réel. C'est à ce même joint où Sinn et Bedeutung s'excluent l'un de l'autre, que se joue, me semble-t-il, la tripartition

13. Lacan, 1959-1960, p. 28-30, et, pour l'application quasi conclusive du terme "impossible" au problème moral, p. 364.

14. "[L'objet $a]$, personne ne sait le voir. (...) Mais que personne ne sache le voir, au sens ou 'sache' c'est 'puisse', n'excuse pas que personne encore n'ait pu le concevoir' (Lacan, 19651966, leçon du 5 janvier 1966).

15. Cf. supra, n. 12. Notons que ce séminaire se conclut sur cette phrase: "C'est dans l'impossible que nous reconnnaissons la topologie de notre désir" (Lacan, 1959-1960, p. 64).

16. Cette autre manière de dire la "sortie" freudienne du narcissisme (Freud, 1914) n'est en effet pas sans renvoyer à l' "admiration” cartésienne (sur ce point, cf. Pellion, 2007). 
entre manque, objet du manque, et agent du manque, qui sous-tend la "théorie" du "manque d'objet" - ou plus exactement du " manque de l'objet" (Lacan, 19561957, p. 37) - dont le Séminaire IV s'essaie à dresser le tableau (Lacan, 1956-1957, p. 9-92). Le point de départ de Lacan, on le sait, est que le manque, en tant qu'éprouvé du sujet, c'est-à-dire fait nucléaire de signification, ne se confond ni avec l'objet dont est attendue réparation de ce manque, ni avec cet autre objet, dit "agent" et à qui sont référées, rétroactivement, l'histoire et l'origine de ce manque. ${ }^{17}$ Mais, au-delà de cette observation, son tour de force est de répartir les trois ${ }^{18}$ "catégories" du "manque d'objet" auxquelles la littérature analytique donne droit de cité selon la manière dont ces trois étages du manque se distribuent entre réel, symbolique et imaginaire. Dans la castration, c'est l'agent qui est à rapporter au réel. Mais, que l'agent de la castration en vienne à être situé ainsi en redouble l'indicibilité de principe, le "parce que c'est comme ça" que l'agent en tant que tel porte en lui-même. ${ }^{19}$

Troisième variation: impossible réalité. Cette spécification du réel rapproche donc l'épreuve de la cause d'une irreprésentabilité. Ce faisant, elle subvertit profondément la manière pour nous coutumière de penser la causalité. Cette manière de penser repose, au fond, sur l'apparente indubitabilité de la formule cartésienne disant qu' "il doit y avoir au moins autant de réalité dans la cause que dans son effet" (Descartes, 1641, p. 298). Le problème de cette phrase étant qu'elle n'est évidente que pour qui la lit comme une mise en rapport, au sens euclidien, de deux choses devant obligatoirement être "du même genre". Car lire Descartes ainsi, c'est tout simplement oublier que cette formule des Méditations, préparée par un

17. Ainsi, quand Alain Vanier définit l'objet transitionnel winnicottien comme "ce qui donne sens de perte au manque" (Vanier), on voit bien que la qualité de la perte se réfère à l'agent. L'important étant alors de suivre quelle valence, réelle, imaginaire ou symbolique, va être conférée par le sujet à cette "perte".

18. Et seulement trois: on peut en effet remarquer que les combinaisons trois à trois (manque, objet, agent) des trois registres (réel, symbolique, imaginaire) sont théoriquement au nombre de $3 !=6$. Privation, frustration et castration ne recouvrent donc qu'une partie - la moitié, précisément - des combinaisons possibles.

19. Bien plus tard, Lacan avouera avoir un peu éludé, dans le Séminaire $I V$, le rôle du dit "agent". Mais c'est pour plaisanter aussitôt sur la régression à l'infini à laquelle il aurait bien pu se trouver conduit: “Qu'est-ce qui fait agir l'agent?” (Lacan, 1969-1970, p. 199). En effet, répondre à cette question reviendrait très exactement à recrédibiliser le fantasme comme récit pas-impossible à comprendre d'une causation (cf. supra)... 
développement extrêmement serré, ${ }^{20}$ identifie de facto, sous l'unique chef de la "réalité", deux choses maintenues soigneusement inégales par des siècles de scolastique: la "réalité formelle", d'une part - visant ce qui entre en concordance avec l'idée dans la chose elle-même, soit la qualité cognitive de l'idée -, et la "réalité objective", de l'autre - faisant référence, celle fois, à ce qui correspond effectivement à la chose dans l'idée, soit à la puissance causale de la chose.

Quatrième variation: identification et impossible. Il me semble donc, au fond, que le trajet qui mène Lacan de la réalité au réel peut aussi se lire comme une analyse de cette identification cartésienne; j'en arrive ainsi à la seconde des dates qui encadrent mon titre, 1962, et au séminaire sur L'identification. Lacan y discute en effet l'écriture suivante, qu'il propose comme formule générale de l'identification, et qui est aussi comme le parangon de l'impossible: "a $\neq a$ " (Lacan, 1961-1962, leçon des 24 janvier et 21 février 1962). Au delà de sa référence frégéenne, ${ }^{21}$ cette écriture reprend - certes sous une forme tellement ramassée qu'elle en devient presque illisible -, le paradoxe freudien quant à "das Ding”: en effet, le "neurone a" ainsi nommé dans l'Esquisse est à la fois le "centre constant" d'où diffuse tout le processus de frayage et la partie qui échappe au "jugement” auquel Freud fait équivaloir ce même frayage (Freud, 1895, p. 341 sqq.).

L'écriture de Lacan viole sans vergogne le principe de non-contradiction, elle est sa négation même: c'est que l'identification, comme envers de la "réalité" cartésienne, opère sur fond d'impossible. Remarquons d'ailleurs que le principe de non-contradiction lui-même s'écrit, originellement, à partir d'une impossibilité. Aristote, dans Métaphysique $G$, le formule en effet ainsi: "Il est impossible - et le

20. Qui culmine, je crois, dans le passage suivant: "On doit savoir que toute idée étant un ouvrage de l'esprit, sa nature est telle qu'elle ne demande de soi aucune autre réalité formelle que celle qu'elle reçoit et emprunte de la pensée ou de l'esprit (...). Or, afin qu'une idée contienne une telle réalité objective plutôt qu'une autre, elle doit avoir cela de quelque cause, dans laquelle il se rencontre pour le moins autant de réalité formelle que cette idée contient de réalité objective" (Descartes, 1641, p. 290, je souligne). En d'autres termes, la plus importante réalité de l'idée est d'être indépendante de la chose dont elle est éventuellement l'idée, parce que cela tient à sa "nature" d'être une idée, c'est-à-dire d'être du registre de la "pensée" ou de l'"esprit", et d'être apte à ce titre à recevoir des distinctions.

21. Gottlob Frege se set en effet de l'écriture inverse, à savoir " $a=a$ a", pour faire valoir l'écart qu'il y a entre sens et dénotation. Si cette proposition n'a aucun sens, il n'en reste pas moins qu'elle n'est pas dépourvue de valeur, puisqu'elle est toujours vraie : sa juste valeur étant alors le vraie en tant qu'une des dénotations possibles de toute proposition complexe (Frege). 
terme $\alpha \delta v v \alpha \tau o v^{22}$ figure en toute lettres dans le texte grec - que le même attribut appartienne et n'appartienne pas en même temps au même sujet et sous le même rapport" (Aristote, 1953, p. 195). Ainsi, la vérité fondamentale de ce qu'on appelle depuis Martin Heidegger "métaphysique occidentale" n'est pas la mise en ordre d'une totalité qui se donnerait dans la concordance, mais bien la dérivation d'une impossibilité causale. ${ }^{23}$

L'impossible se loge donc à cette place du hiatus causal que la mise en rapport cartésienne des effets et des causes a voulu compter pour rien; et, de cette place tout à fait singulière, exceptionnelle, ${ }^{24} \mathrm{c}$ 'est bien à lui qu'il revient de "préserve[r] [c]es droits du rien" (Lacan, 1961-1962, leçon du 7 mars 1962) qui donnent, en 1962, son plein statut au "rien de réel" de 1951. C'est dire en peu de mots l'accès exprès au réel qu'il autorise, et sa contribution à la "science du désir" (Lacan, 1958-1959, leçon du 12 novembre 1958).

\section{$*$}

Je crois qu'on peut conclure en disant deux choses. Premièrement que, pour Lacan, on ne parle valablement que $d u$ réel. En précisant toutefois que ce "du" est à entendre non seulement au sens objectif de la science - qui formalise un objet réel en tant qu'impossible à rejoindre par la sensibilité, si ce n'est asymptotiquement -, mais également au sens subjectif d'un "à partir du" réel. Témoigne de cela, par exemple, ce passage de "Position de l'inconscient", où Lacan reprend l'expression de "sujet réel", déjà utilisée antérieurement - par exemple dans le séminaire sur Les formations de l'inconscient (1957-58)-, mais avec un

22. Adunatos a comme sens premier, en grec, incapable. On voit l'antiquité du glissement lacanien de l'impuissance à l'impossible !

23. Ceci se vérifie d'ailleurs en bien d'autres endroits de la tradition philosophique que dans le seul texte d'Aristote. Ainsi, pour ne prendre que ce seul exemple, et qu'il faudrait développer avec plus de détail, dans cette formulation ultime du cogito cartésien, dont on voit sans peine ce qu'elle doit encore, dans sa lettre, au principe aristotélicien: "Mais nous ne saurions supposer de même que nous ne sommes point tandis que nous doutons de la vérité de toutes ces choses; car nous avons tant de répugnance à concevoir que ce que qui pense n'est point au même temps qu'il pense, que, nonobstant toutes les plus extravagantes suppositions, nous ne saurions nous empêcher de croire que cette conclusion: Je pense, donc je suis, ne soit vraie, et par conséquent la première et la plus certaine qui se présente à celui qui conduit ses pensées par ordre" (Descartes, 1644, p. 573).

24. Dans la leçon inédite du 7 mars 1962 du séminaire sur L’identification, Lacan montre comment l'universel du nécessaire dérive, à proprement parler, de la position d'un impossible ("il est impossible qu'un trait ne soit pas vertical" précède logiquement "tout trait est vertical" car donne droit à l'ensemble vide...). 
tout autre sens: "L'effet de langage, c'est la cause introduite dans le sujet. Par cet effet il n'est pas cause de lui-même, il porte en lui le ver de la cause qui le refend. Car sa cause, c'est le signifiant sans lequel il n'y aurait aucun sujet dans le réel" (Lacan, 1964b, p. 835).

Deuxièmement, que l'impossibilité inhérente à la position analytique est corrélative de la proximité du réel par laquelle elle se spécifie (Assoun). Proximité dont il n'y a, bien sûr, en tant que proximité, aucun critère mesurable, ni probablement aucune déduction représentable.

\section{Références}

Aristote. Métaphysique. Tr. fr. Jean Tricot. Paris: Vrin, 1953.

Assoun, P.-L. Du métier impossible à l'acte nécessaire. Figures de la psychanalyse, Paris, n. 15, p. 16-31, 2007.

Cotard, J. Intervention du 28 mars 1887 à la Société Médico-Psychologique. Ann. Méd.-Psychol., Paris, p. 57-80, 1887.

Cuse, N. de. De la docte ignorance. Tr. Paris: La Maisnie, 1979.

Descartes, R. (1641). Méditations. Tr. fr. in Euvres et lettres. Paris: Gallimard, 1953. p. 257-547. (Pléiade)

. (1644). Les principes de la philosophie. Tr. fr. in Euvres et lettres. Paris: Gallimard, 1953. p. 549-690. (Pléiade)

FREGE, G. Sens et dénotation. Tr. fr. in: Écrits logiques et philosophiques. Paris: Seuil, 1971. p. 102-126.

Freud, A. Le moi et les mécanismes de défense. Tr. fr. Paris: PUF, 2001.

FREUD, S. (1895). Esquisse d'une psychologie scientifique. Tr. fr. in La naissance de la psychanalyse. Paris: PUF, 1956. p. 307-396.

. (1912). Des types d'entrée dans la maladie névrotique. Tr. fr. in Euvres complètes. Paris: PUF, 1998. t. XI, p. 117-126.

. (1914). Pour introduire le narcissisme. Tr. fr. in Euvres complètes. Paris: PUF, 2005. t. XII, p. 217-245.

. (1915). Le refoulement. Tr. fr. in Euvres complètes. Paris: PUF, 1988. t. XIII, p. 187-201. . (1919a). "Un enfant est battu" - Contribution à la connaissance de la genèse des perversions sexuelles. Tr. fr. in Euvres complètes. Paris: PUF, 1996. t. XV, p. 115146. 
. (1919b). L'inquiétant. Tr. fr. in Euvres complètes. T. XV. Paris: PUF, 1996. t. XV, p. $147-188$.

. (1921). Psychologie des foules et analyse du moi. Tr. fr. in Euvres complètes. Paris: PUF, 1991. t. XVI, p. 1-83.

. (1924a). Névrose et psychose. Tr. fr. in Euvres complètes. T. XVII. Paris: PUF, 1992. t. XVII, p. 1-7.

. (1924b). La perte de la réalité dans la névrose et la psychoses. Tr. fr. in Euvres complètes. Paris: PUF, 1992. t. XVII, p. 35-41.

Gori, R. Logique des passions. Rééd. Paris: Champs/Flammarion, 2005.

Guéroult, M. Descartes selon l'ordre des raisons. Paris: Aubier Montaigne, 1968.

Hartmann, H. La psychologie du Moi et le problème de l'adaptation. Tr. fr. Paris: PUF, 1968.

LACAN, J. (1936). Au-delà du principe de réalité. In Écrits. Paris: Seuil, 1966. p. 73-92.

226.

. (1951). Intervention sur le transfert. In Écrits. Paris: Seuil, 1966. p. 215-

juillet.

(1953). Le symbolique, l'imaginaire et le réel. Conférence inédite du 8

. (1953-54). Le séminaire. Livre I. Les écrits techniques de Freud. Paris: Seuil,

1975.

. (1954-55). Le séminaire. Livre II. Le moi dans la théorie de Freud et dans la technique de la psychanalyse. Paris: Seuil, 1978.

. (1955-56). Le séminaire. Livre III. Les psychoses. Paris: Seuil, 1981.

. (1956-57). Le séminaire. Livre IV. La relation d'objet. Paris: Seuil, 1994.

. (1957). L'instance de la lettre ou la raison depuis Freud. In: Écrits. Paris:

Seuil, 1966. p. 493-528. Seuil, 1998.

(1957-58). Le séminaire. Livre V. Les formations de l'inconscient. Paris:

. (1958a). D'une question préliminaire à tout traitement possible de la psychose. In: Écrits. Paris: Seuil, 1966. p. 531-583.

$6 \overline{95 .}$

. (1958b). La signification du phallus. In: Écrits. Paris: Seuil, 1966. p. 685-

. (1958-59). Le séminaire. Livre VI. Le désir et son interprétation. Inédit, sauf les leçons consacrées à Hamlet, parues in Ornicar?, n. 24, p. 7-31, 1981; n. 25, p. 13-36; n. 26-27, p. 7-44, 1982. 
. (1959-60). Le séminaire. Livre VII. L'éthique de la psychanalyse. Paris: Seuil, 1986.

. (1961). La direction de la cure et les principes de son pouvoir. In: Écrits. Paris: Seuil, 1966. p. 585-642.

. (1961-62). Le séminaire. Livre IX. L’identification. Inédit.

. (1964a). Le séminaire. Livre XI. Les quatre concepts fondamentaux de la psychanalyse. Paris: Seuil, 1973.

. (1964b). Position de l'inconscient. In: Écrits. Paris: Seuil, 1966. p. 829-850.

. (1965-66). Le séminaire. Livre XIII. L'objet de la psychanalyse. Inédit.

724. . (1966). D’un syllabaire après coup. In: Écrits. Paris: Seuil, 1966. p. 717-

. (1968). Proposition du 9 octobre 1967 sur le psychanalyste de l’École. In:

Autres écrits. Paris: Seuil, 2001. p. 243-259.

. (1969). L'acte psychanalytique - Compte rendu du séminaire 1967-1968. In: Autres écrits. Paris: Seuil, 2001. p. 375-383.

. (1968-69). Le séminaire. Livre XVI. D'un Autre à l'autre. Paris: Seuil, 2006.

Seuil, 1991.

. (1969-70). Le séminaire. Livre XVII. L'envers de la psychanalyse. Paris:

. (1975-76). Le séminaire. Livre XXIII. Le sinthome. Paris: Seuil, 2006.

Pascal, B. Infini rien. In: Pensées. Euvres complètes. Paris: Gallimard, 1954. p.

1212-1216. (Pléiade)

Pellion, F. (2004-2007). Histoire de l'a - Une hésitation de Jacques Lacan.

Séminaire de recherche. Association CoRA, Collège Clinique de Paris (Formations

Cliniques du Champ lacanien), équipe d'accueil "Médecine scientifique, psychopathologie et psychanalyse" de l'école doctorale "Recherches en psychanalyse" (université Paris VII - Denis Diderot).

. (2007). Figures cartésiennes de 1' "exclusion interne". Cliniques

Méditerranéennes, Paris, n. 76, p. 207-216, 2007.

VANIER, A. Mouvements de l'objet. Mensuel (ÉPFCL - France), Paris, n. 18, p. 2030, 2006.

WinnicotT, D. W. Objets transitionnels et phénomènes transitionnels. In: De la pédiatrie à la psychanalyse. Paris: Payot, 1969. p. 169-186. 


\section{Resumos}

(Jacques Lacan, para o real [1936-1962])

A construção de Jacques Lacan da categoria do "real", mais do que as do "simbólico" e do "imaginário", não foi sem dificuldades e sem arrependimentos. Aqui, trata-se da questão dessas dificuldades, de algumas etapas de suas posições e finalmente, de suas resoluções, esperando, no final do percurso, poder talvez situar um pouco melhor o papel dessa categoria na "metapsicologia" lacaniana - sobretudo se admitimos que esta pode interagir com as metapsicologias vizinhas, ainda mais com numerosos aspectos do "dizer" filosófico.

Palavras-chave: Impossível, objet a, sujeito, verdade

(Jacques Lacan hacia lo real [1936-1962])

Mas que las categorías de simbólico y de imaginario, la construcción de Jacques Lacan de la categoría de real no fue hecha sin dificultades ni arrepentimientos. Son esas dificultades y algunas de las etapas de su posicionamiento y resolución lo que pondremos en cuestión aquí; no sin esperar, al término de este recorrido, poder situar mejor el rol de lo real en la metapsicología lacaniana - sobretodo si admitimos que esa categoría está en interacción con las metapsicologías vecinas y con numerosos aspectos del "decir" filosófico.

Palabras claves: Imposible, objeto a, sujeto, verdad

(Jacques Lacan towards the real [1936-1962])

Jacques Lacan's construction of the category of the "real" brought with it more difficulties and doubts than did his categories of the "symbolic" and the "imaginary." This article discusses these difficulties together with certain steps he took concerning their introduction and later resolution. The aim of the investigation is to better situate the role of this category of the "real" within Lacan's "metapsychology" - especially since we acknowledge that this metapsychology necessarily interacts with neighboring metapsychologies, as it also interacts with various aspects of the philosophical "act of saying."

Key words: Impossible, object petit a, subject, truth

Citação/Citation: Pellion, F. Jacques Lacan vers le réel (1936-1962). Revista Latinoamericana de Psicopatologia Fundamental, São Paulo, v. 12, n. 1, p. 99-115, mar.2009.

Editor do artigo/Editor: Prof. Dr. Manoel Tosta Berlinck.

Recebido/Received: 13.8.2008 / 8.13.2008 Aceito/Accepted: 20.8.2008 / 8.20.2008 
Copyright: @ 2009 Associação Universitária de Pesquisa em Psicopatologia Fundamental/ University Association for Research in Fundamental Psychopathology. Este é um artigo de livre acesso, que permite uso irrestrito, distribuição e reprodução em qualquer meio, desde que $\mathrm{o}$ autor $\mathrm{e}$ a fonte sejam citados/This is an open-access article, which permits unrestricted use, distribution, and reproduction in any medium, provided the original author and source are credited.

Financiamento/Funding: $\mathrm{O}$ autor declara não ter sido financiado ou apoiado/The author has no support or funding to report.

Conflito de interesses: $\mathrm{O}$ autor declara que não há conflito de interesses/The author declares that has no conflict of interest.

\section{Frédéric Pellion}

Docteur en médecine et en sciences humaines cliniques (psychopathologie fondamentale et psychanalyse). Psychiatre, praticien hospitalier, Centre hospitalier SainteAnne; psychanalyste; enseignant (HDR) à l'université Paris VII - Denis Diderot (Centre de Recherche Psychanalyse et Médecine), à l'université Paris V - René Descartes et au Collège clinique de Paris; membre de l'École de psychanalyse des Forums du Champ lacanien.

25 , rue Tournefort 75005 Paris, France

e-mail: f.pellion@wanadoo.fr 